\title{
Relating Corroded Seven-Strand, Posttensioned Cable Cross-Sectional Properties to Load Capacity
}

\author{
Richard Haskins, Barry White, Robert Ebeling, and James Evans \\ U.S. Army Engineer Research and Development Center, 3909 Halls Ferry Rd., Vicksburg, MS 39180, USA \\ Correspondence should be addressed to Richard Haskins; richard.w.haskins@usace.army.mil
}

Received 21 March 2016; Accepted 20 September 2016

Academic Editor: Lucio Nobile

Copyright ( 2016 Richard Haskins et al. This is an open access article distributed under the Creative Commons Attribution License, which permits unrestricted use, distribution, and reproduction in any medium, provided the original work is properly cited.

\begin{abstract}
Multistrand anchors have seen widespread use, providing strength and stability at hydraulic Corps facilities. However, these steel tendons are subject to strength reduction as an effect of corrosion. Methods for evaluating the corroded cable strength do not exist to accurately estimate the time until tendon cables would have to be replaced (at great expense). The following five research tasks are used to address this deficiency: laboratory accelerated corrosion; pull-tests on pristine and laboratory corroded cables; optical scanning; data collection correlated with cross-sectional properties of cables; and development of a method to relate this data to the field. The pull-tests provide measured capacities for seven-strand, posttensioned (PT) cables. An optical scan of the corroded cables provides cross-sectional properties of individual wires within the pulled cables. Trendlines are established for the related peak cable capacities and cross-sectional properties in an effort to determine their correlations. Trendlines for minimum wire area and second-moment short axis diameter are found with low error, making them good predictors of loaded cable capacity. This pull-test dataset has been related back to cable failure in the field, assuming a linear rate of corrosion loss for the cross-sectional properties and required PT capacity.
\end{abstract}

\section{Introduction and Background}

Over the past five decades, the U.S. Army Corps of Engineers has installed numerous high-capacity concrete posttensioning systems using tendons comprised of several grouted seven-strand cables. These stressed steel tendons have been used to strengthen hydraulic structures and to improve their serviceability and stability. A seven-strand cable has sixstrand wound about a larger diameter center strand called the king wire.

While substantial improvements in multistrand anchorsystem corrosion protection have occurred progressively over the years, the corrosion of older installations is a major area of concern. This is due to the inferior corrosion-protection mechanisms at the time of installation and increased exposure times. Typically, there is little or no visible evidence of ongoing subsurface corrosion for these anchor systems. Given the high-tension nature of these systems, rapid and unforeseen structural failure is a real likelihood. This article investigates corrosion-based material losses and seeks to better define their influence on typical seven-strand, posttensioned (PT) cable systems.

1.1. Limitations of Current Assessment Technologies. Ideally, what is needed is a quantitative, nondestructive inspection method capable of providing in situ characterization of the ongoing corrosion rate and remaining cable material. For embedded PT cable installations, no suitable method currently exists. The typical navigation structure anchorage has only one end of the cable accessible without either shallow or parallel access through the cable's concrete cover. A 2012 Federal Department of Transportation report [1] provides a comprehensive assessment of a wide range of inspection technologies on controlled bridges with the goal of evaluating seven-strand inspection capabilities and performance. Inspection needs were divided into several groups based on the condition being targeted: breaks and cross-sectional loss, improper grout condition, active corrosion, tension, and duct location and condition. In general, the investigated methods rely on having cable access from both ends, shallow concrete 
cover, or exposed tendon ducts. As these conditions do not generally hold for mass concrete retrofits and navigationbased structural elements, the authors believe that acoustic emission, modified electrical corrosion assessment methods, and ultrasonic guided wave technologies [2] represent the most promising Nondestructive Testing (NDT) methods for future assessment of PT cable of navigation structures.

In the case of corrosion model development, it is clear that the accuracy and performance of any condition estimation method are directly tied to the capability to accurately quantify critical in situ conditions. Estimating the inputs that determine corrosion rate, such as chloride content, voids in grout, moisture presence, and macrocell potentials, is difficult due to the same accessibility constraints creating difficulty with NDT method development.

1.2. Corrosion Damage of Embedded Strands. The corrosion process of prestressed steel tendons consists of two phases: corrosion initiation, usually occurring before the cable is embedded, and corrosion propagation, informing the rate of corrosion, which will determine the life span of the cable [3]. Corrosion actually initiates when the protective oxide film that has formed around the steel member is compromised by a chloride concentration that exceeds a threshold or by lowering the $\mathrm{pH}$ of the grout [4]. At least seven types of corrosion mechanisms are involved in prestressed anchorage corrosion: pitting corrosion; uniform corrosion; stress corrosion cracking; hydrogen cracking; crevice corrosion; fretting corrosion; and fatigue corrosion [5]. All are invasive to the material. Pitting corrosion has the most effect on cable capacity because it changes the cross-sectional area of the cable wires rapidly. Some of these methods of corrosion are not likely to occur in PT anchorage systems. Stress corrosion cracking is primarily a concern for very old PT anchorages where the annealing process created susceptibility to failure due to increased hydrogen [6]. Likewise, crevice corrosion and corrosion due to fungal and mold growths in the PT grease are generally considered less of a deterioration concern than that of pitting corrosion. In most cases, corrosion is an electrochemical reaction and acts in cells like batteries, where cathodes corrode and transfer electrons to anodes.

Corrosion of strand anchorages occurs in many forms and is influenced by several factors such as temperature, oxygen concentration, water presence, salts and/or acids present, grout electrical resistance, $\mathrm{pH}$, microbial activity, and scaling tendency. From the number of these factors, it can be deduced that any method chosen to protect the tendons must be able to protect the strands from a myriad of these influences at all times. In practice, older unbonded tendons are protected by anticorrosive grease surrounding the cable in a polymer sheath, which is then surrounded by a protective grout. In even older installations, only a protective grout may be present around the cable with no protective plastic or metal PT duct. Due to proximity to environmental moisture ingress, stress concentration at the anchor wedges themselves, and the absence of any redundant protection at the grip mechanism, the anchor-head region tends to be a region of high probable failure within the cable PT system. Macrocell corrosion as opposed to microcell corrosion is another major concern of
PT cable corrosion. The larger macrocell corrosion cells are more likely to occur between regions where the embedded part of the cable serves as the cathode and the grout interface region near the anchor head serves as the anode [4]. The macrocells conduct current along a larger reach of the anchor. If the macrocell current density is the same as a microcell, the microcell corrosion damage will be greater, having the same volume of damage over a more localized area of the cable.

One of the primary causes of tendon failure in anchor assemblies is the failure of the grout protecting the tendons. Voids occurring at the time of construction allow moisture to penetrate into the PT duct and corrode the cables. The absence of the grout also eliminates the high-pH surface passivation, which serves to protect the cable. Various studies on tendon-failure cases in bridges also indicate that the presence of chlorides in grouts is a major contributor to tendon failure [7]. Unlike bridges where ocean or brackish saltwater and deicing salts produce environmental-based increases in concrete and grout chlorides, navigation structures are not generally expected to suffer increased chloride levels with time. In general, if high-grout chlorides are present in navigation structures, it would be due to problems occurring in the initial batching operations.

Pipelines, boiler tubes, and storage tanks lend themselves well to the development of corrosion models for condition estimation. In these structures, the properties and conditions of the materials in contact with the steel are easily quantifiable and the systematic attack on the material can be reasonably estimated. Even though we know the various mechanisms that affect or facilitate deterioration of PT cables, these mechanisms tend to occur randomly and not systematically. Without direct access, any estimation of corrosion in PT cables can have a significant degree of uncertainty.

\section{Laboratory Specimen Testing and Analysis}

A laboratory correlation method was developed to relate the cross-sectional properties of deteriorated seven-strand cables near their break locations to their ultimate capacity. The cables used in these laboratory experiments were low relaxation, $15.24 \mathrm{~mm}$ (0.6 in.) diameter cables of 1,861.6 MPa (270 ksi) capacity, which are typical for anchorage installations. A controlled accelerated corrosion process was used in an effort to match the natural corrosion damage seen in recovered strands. A corrosion-based material loss is inherently difficult to characterize due to nonuniform losses such as large surface pitting. To solve the challenge of corrosion loss characterization, an optical method was developed based on morphological image analysis at the break location after a pull-test.

2.1. Inducing Corrosion in Laboratory Cable Specimens. A corrosion system was constructed that used a destructive electrochemical process to liberate iron from the steel's surface. A salt solution served to both carry the electrical potential and introduce destructive chlorides. Heat and aeration were added as catalysts to further accelerate the process. An accelerated corrosion system was constructed consisting of the following components: brine electrolyte solution made of 


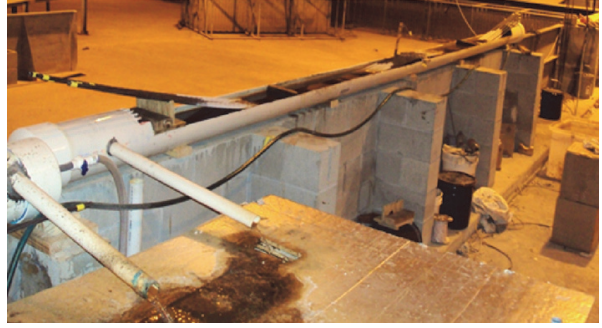

(a)

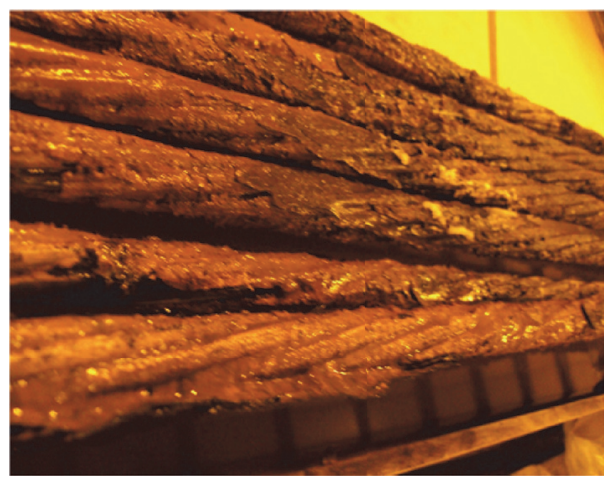

(c)

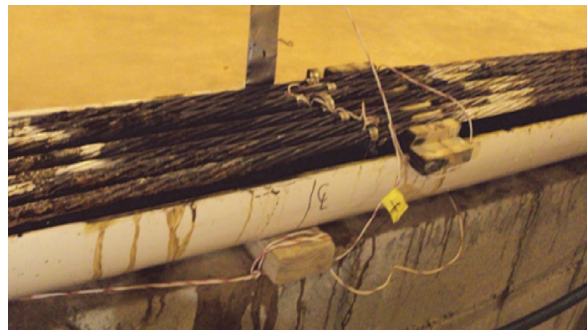

(b)

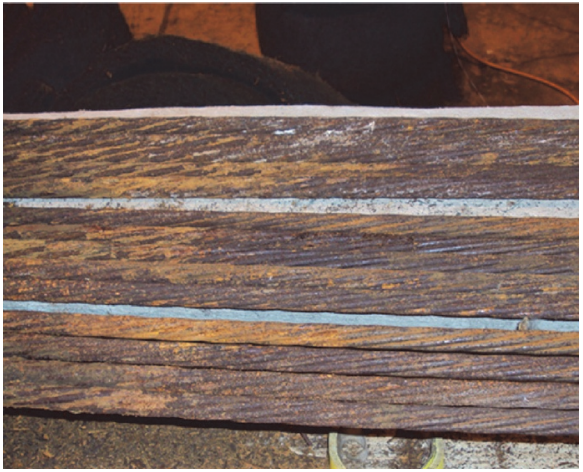

(d)

FIGURE 1: Photographs of corrosion tanks and corroded seven-strand cables.

tap water and salt $(\mathrm{NaCl})$; direct-current power supply with positive lead attached to cables and negative lead attached to submerged steel grid (anode); spacer to separate cables and anode from contact; water heater; reservoir tank with brine solution; and a pump for circulation and aeration. A second accelerated corrosion system was constructed with only the first three components to see the effect of static fluid conditions. The second system produced less uniform corrosion with more pitting.

In some of the more severely corroded cables, nonuniform corrosion was observed. This nonuniform corrosion manifested as a deeper pitting on one side of the cable, sometimes near midspan, but more commonly at the waterline. The midspan pits, thought to be a result of iron buildup in the bottom of the cable tank, were addressed by more frequent inspections and cleanout. The waterline pits were thought to be a result of salt precipitation and higher oxygen levels. These waterline pits were handled by protecting the cable in this area with a layer of silicone caulk. Initial investigations for corrosion system development indicated that the circulation process produced relatively smoother deterioration than that of the static tanks with high salt concentrations (precipitated). Figures 1(a) and 1(b) show two different views of one of the cable tanks. Figures $1(\mathrm{c})$ and $1(\mathrm{~d})$ show cables just removed from the bath (c) and after drying (d).

2.2. Pull-Test to First Wire Failure with Pristine and Laboratory Corroded Specimens. Mechanical testing using a Baldwin pull-test machine obtained stress-strain curves for deteriorated specimens pulled from the corrosion chambers. An example set of stress-strain curves is shown in Figure 2. Standard PT grip wedges were used on the deteriorated

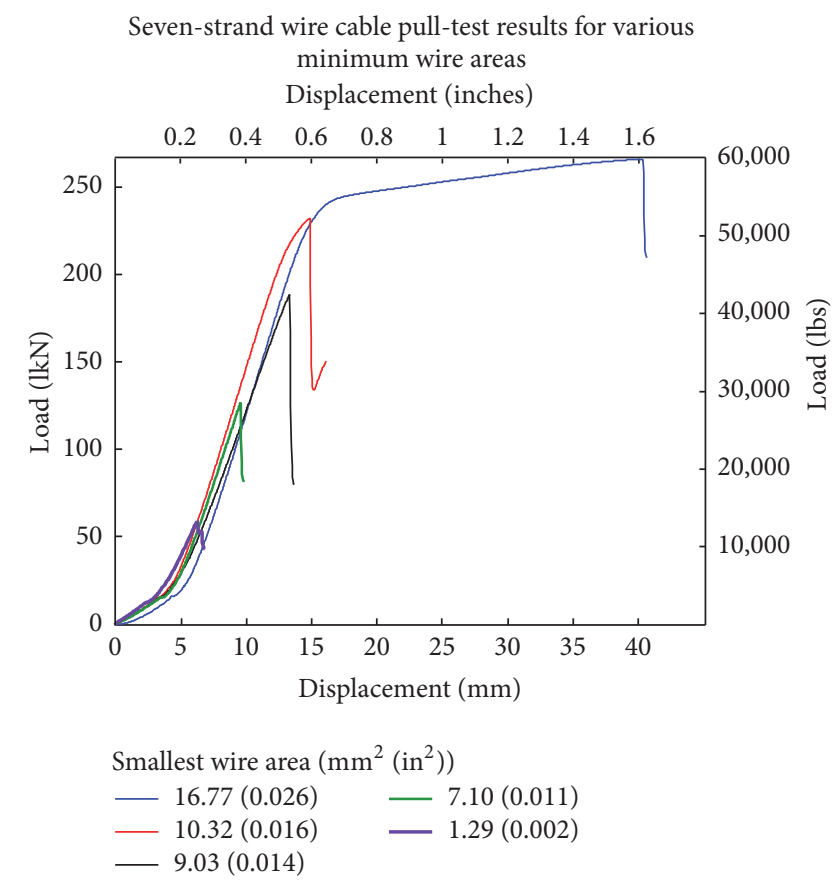

FIGURE 2: Example pull-test results from various deteriorated cables.

specimens, and specialized grip mechanisms were used to obtain data for pristine cables.

The pristine (i.e., uncorroded or undamaged) cables were needed for comparison purposes in the corrosion damage assessment. Because the grip wedge represents a stress concentration point that literally bites into the cable, grip wedges 


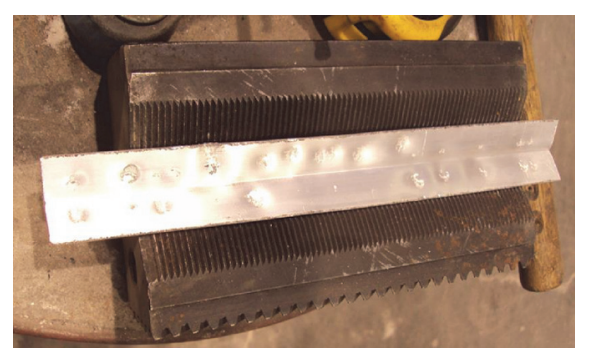

FIGURE 3: Hammering aluminum strips into the wedge to form grip points with serrations.

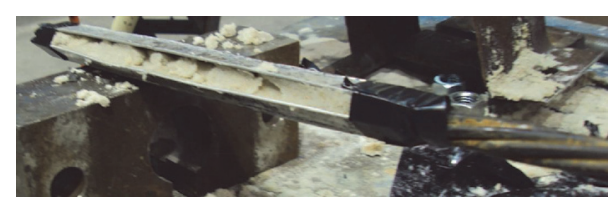

FIgURE 4: Detail of the cables being restrained with the aluminum strips and wet sand mixture.

cause and localize most failures in a pristine cable. The wedge is known to decrease the ultimate capacity by approximately $5 \%$. Several gripping methods for pristine cable were attempted that did not overcome this stress concentration point problem.

The final method chosen for testing pristine cables without stress concentration at the wedges was based on Moser [8]. This approach uses a light sand mixture formed around the cable and confined with two aluminum strips formed into the shape of the wedges. Two $38 \mathrm{~mm} \times 203 \mathrm{~mm} \times 3.2 \mathrm{~mm}$ (1.5 in. $\times 8$ in. $\times 0.1259$ in.) aluminum strips were shaped to the form of the large wedges seen in Figure 3. Hammering the aluminum strips into the wedges caused the scalloped indentations in the wedge to indent the aluminum, strengthening the grip of the aluminum strips within the wedge. Then, light wet sand was mixed and formed around the cable. The aluminum pieces were placed to compress the sand around the cable. The aluminum pieces were tightened using cable ties and reinforced with electrical tape (Figure 4). This method for gripping the pristine cable produced consistent breakage in the middle part of the cable (away from the wedges). In this case, the end length of cable that was gripped by the aluminum and sand enclosure was nearly $280 \mathrm{~mm}$ (11 in.). This procedure was then used successfully on a total of 22 pristine cable specimens.

The 22 pristine pull-tests resulted in PT seven-strand cable capacity values ranging from a low value of $266.715 \mathrm{kN}$ $(59,960 \mathrm{lbf})$ to a high value of $278.121 \mathrm{kN}(62,524 \mathrm{lbf})$. The computed mean force for separation of the pristine PT seven-wire strand cable is $274.451 \mathrm{kN}(61,669 \mathrm{lbf})$, with a standard deviation for the samples being $2.687 \mathrm{kN}$ (604 lbf). The coefficient of variation (COV) equals 0.0098 . Figure 5 shows a histogram of the pull-test results binned in 13 bins starting at a low number of $266.004 \mathrm{kN}(59,800 \mathrm{lbf})$, with each bin range being $1.334 \mathrm{kN}$ (300 lbf). The probability density function is tallied by taking the number of samples that were

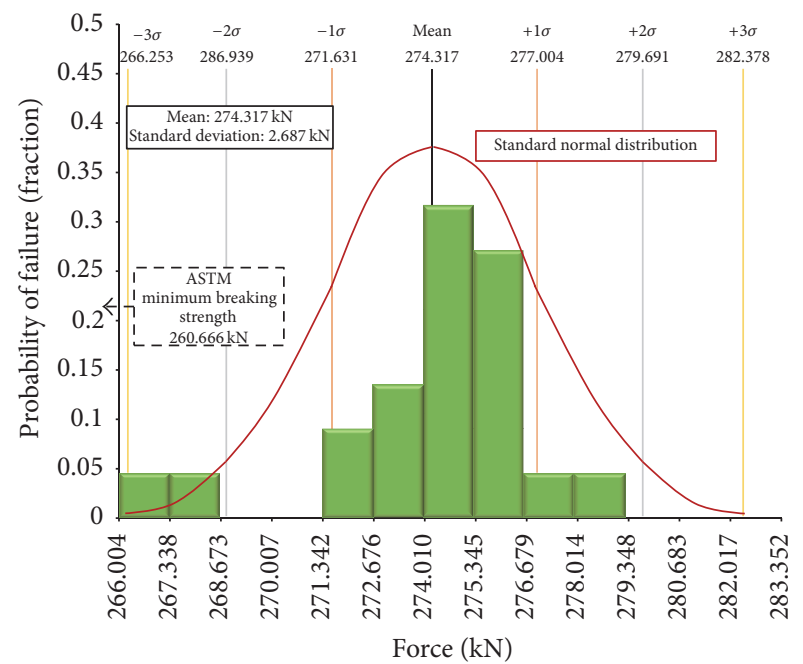

FIGURE 5: Statistical distribution data of failure strength for 22 successful pristine wire tests with a mapped normal distribution for comparison.

counted in each bin range and dividing by a total number of 22 specimens. Observe that the American Society for Testing and Materials (ASTM) minimum breaking strength is exceeded by all 22 cables. Figure 5 also displays the mean and values for three standard deviations above and below the mean. Mapping a standard normal distribution to the data reveals that the data roughly follows that distribution.

2.3. Optical Scanning. The pull-tests provided the pristine and corroded seven-strand cable capacity for individual cables. In order to determine the effects of corrosion, the remaining cross section properties of each cable need to be assessed. This section describes the morphological imaging process used to characterize material losses due to corrosion at the point along the cable where the cable broke.

The following procedure for preparing broken cables provides a way to capture reasonably accurate cross-sectional properties of each broken cable near its failure point. Typically, one to three wires will rupture at failure. The wires that exhibit ductile necking are considered the initial failures and the other failed wires are thought to break due to the sudden release of potential energy and the remaining load level being redistributed. In some cases, a number of the wires in the cable will not fail. After failure, the broken wires will typically "birdcaging" or deform inelastically away from the failure point. This can happen on one or both sides of the failure point. When both sides birdcaging, it is necessary to retrace the separated wires back to the original point of failure so that the remaining wires in the seven-strand bundle can be cut at the appropriate location. Wires are clamped with a small U-bolt type cable fixture prior to cutting in order to maintain proper alignment. Cables are cut just beyond any ductile necking region which is typically less than $2 \mathrm{~mm}$ (0.0787 in.) and orthogonal to their original lay orientation. A cut-off saw is used to perform the initial cutting of the seven-wire strands. The cut ends of the strands are then sanded to provide a smooth surface for optical scanning. 


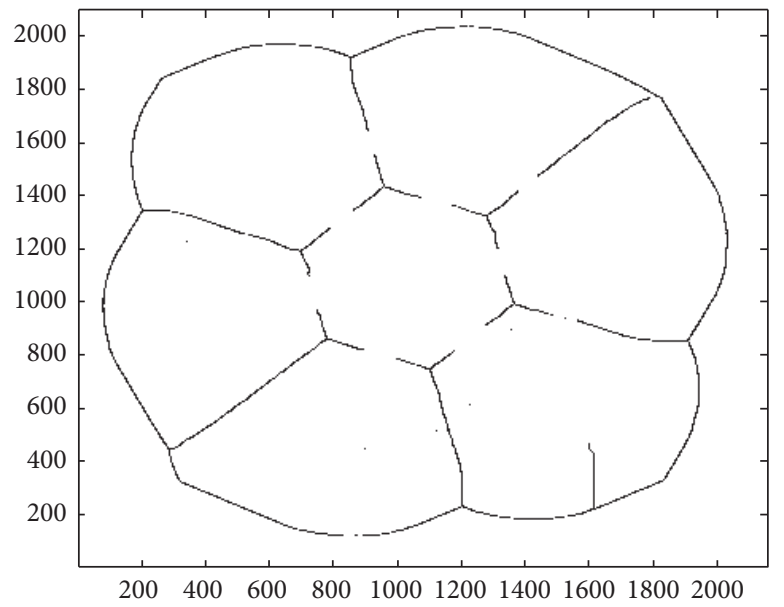

(a)

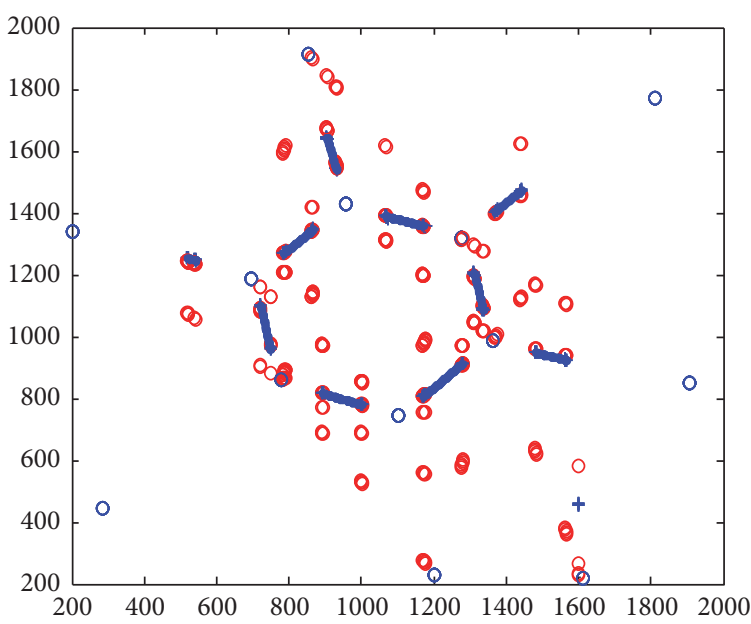

(b)

FIGURE 6: (a) Left: noncontinuous lines where wire objects are touching; (b) right: blue line segments used to separate touching objects for morphological processing.

The cut and processed strand is loaded in a mounting rig that optimizes alignment with the camera for optical scanning and avoids the capturing of and focusing on the undesired background components. After adjusting focus and alignment, a calibration dot is placed on the end of the cable as shown in Figure 3.2 of Ebeling et al. [3]. This dot has a known dimension. An image capture of the dot allows for the automatic generation of a calibration scale between observed pixels and the dot's known dimensions. After removing the calibration dot, lighting via a small LED light is adjusted to best illuminate the end of the cable while minimizing background that results from the helical twist of the cable. A light coat of white paint, applied to the cut end of the cable, was used to improve the contrast between the desired surface and the background. The images were postprocessed using a U.S. Army Engineer Research and Development Center(ERDC-) developed morphological analysis code (briefly described below) and the MATLAB image-processing toolbox, a product of MathWorks, Inc. The postprocessed results provided the individual wire properties of area, perimeter, eccentricity, long axis length, and short axis length. The long axis and short axis length were captured from the second area moment analysis of the wire imagery. The statistical properties of the seven wires (mean, total, and standard deviation) were collected, as well as a labeled image where the strands are numbered in order of decreasing area and mapped to their geometrical measurements. These properties were imported into a database to provide future mechanisms for analysis of the cables performance. The repeatability of the system is greater than $99 \%$, and the length error after calibration is less than $0.08 \mathrm{~mm}$ ( $0.0031 \mathrm{in}$.). The following sections describe in detail the steps and process required in going from the broken cable to the desired geometric properties. The image analysis operation was performed for the cable on both sides of failure point, which produced a second dataset for each broken cable.

Separating touching objects for image analysis is a common and difficult problem in image processing [9]. The common method of watershed analysis was attempted under several preprocessing approaches without achieving adequate and robust performance. Additionally, other approaches based on dilation and skeletonization showed promise but proved to be too slow and performed poorly when less-thanperfect images were presented. For this study, a new approach has been developed. This approach has been tested and shown to be robust across a wide variety of input conditions. This approach is applicable to touching objects which are round and nonoverlapping. The goal of this routine was to generate binary cut lines between touching objects which cannot be separated by simple methods such as edge enhancement and grayscale thresholding.

To generate an image-cutting mask, the captured grayscale image is converted to a binary image, inverted, and infinite thinning of the structures is performed. This results in the image shown in Figure 6(a), where the disconnected line segments in this image represent regions where the objects are touching each other and cannot be morphologically segmented. The objective is to robustly connect these disconnected "lines" and then use them as a binary mask for cutting the touching objects. The next step is to run a routine that removes spurs. Spurs are open line segments such as those appearing near the points where the individual strands touch. If spur removal is run iteratively, the difference in two image iterations can be used to find the points that need connecting in the image mask for segmentation. In the next step, branch points are identified. Branch points are points where three or more lines come together. The fact that the missing mask segments (and spur points) are approximately in a line with the detected branch points forms the basis for the mask generation process described below.

A statistical-based search routine is executed, finding the closest six neighbors for each branch point in the image. A normalized regional spur points error equation was developed, which summed the distances from the line and divided the total by the spur points contained between the $x$ and $y$ 


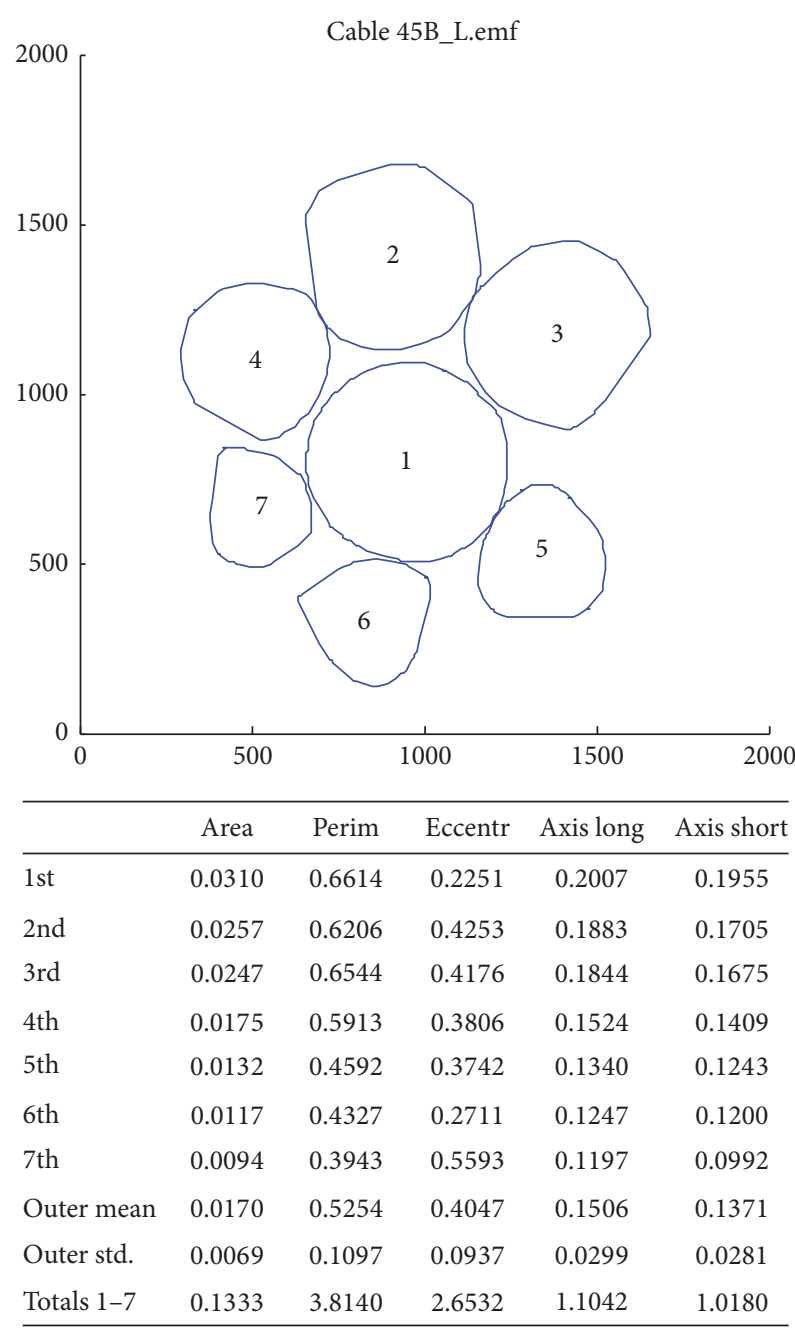

FIGURE 7: Length and area analysis for each wire (units in inches and square inches: conversion to $\mathrm{mm}$ and $\mathrm{mm}^{2}$ by multiplying by 25.4 and 645.16 , resp.).

limits between the branch points. If the error value is less than 1000, then the points are considered proper and a line, continuous in $x$ and $y$ pixels, is formed between the spur points.

Adding these missing segments (shown in Figure 6(b)) creates the complete image mask needed for segmenting the seven strands into unique objects. The amount of area lost in the masking process is considered insignificant with relation to the total wire area.

After proper segmentation and calibration, morphological functions in the MATLAB image-processing toolbox were used to develop measurements of area, length, eccentricity, and second-moment minimum and maximum diameters for each of the wires across the entire corrosion/pull-test dataset. Figure 7 shows an example dataset for a cable imaged at the failure point.

Figure 7 shows the cross section and lists the properties of the corroded cable 45B_L. In this figure, the strand labeled 1 is the king wire and it is surrounded by 6 outer wires labeled 2 through 7. Each strand experienced different levels of corrosion loss. The remaining cross section area of the entire cable is $86 \mathrm{~mm}^{2}$, as compared to the pristine cable area of $140.28 \mathrm{~mm}^{2}$. The most corroded strand is the wire labeled 7 , with a short axis diameter of $2.52 \mathrm{~mm}$ and an area of $6.06 \mathrm{~mm}^{2}$.

2.4. Collection and Analysis of Pull-Test Results. A total of 183 pull-tests were investigated to determine the relationship between ultimate force at the point of failure of the cable and the optically scanned, cross section morphological data, which was collected for the corroded samples and assumed for the pristine cases. The cross section cable data were analyzed against the pull-test results to find which entities were strongly correlated to ultimate tensile strength. The cross section data included combinations of properties which might indicate a system-like response, such as total area of the two and three smallest wires, the product of the total wire area and smallest wire area, and the product of eccentricity with minimum second-moment diameter. The motivation for these investigations was to explore the potential of a synergistic response due to the cable's helical geometry about the king wire. No such effect was observed and the cable's tensile strength was ultimately best mapped to either the minimum second-moment short axis diameter or minimum area of the smallest wire in the seven-strand assembly. For the discussions below, any mention of diameter will be referring to the short axis second-moment diameter. This result indicates that a single wire can affect the capacity for an entire cable and represents a valid failure model for the cable.

Although there is some spread in the corroded data and even a little spread in the pristine data, there is a noticeable functional correlation between the peak force and both the minimum wire area and the minimum wire short axis diameter in the cable. The cable's capacity increases as the minimum wire area and minimum wire diameter increase, which was expected. Statistically, the trend of the data-fit could be determined by using a least-fit method with different forms of equations using the coefficient of determination $\left(R^{2}\right)$ measure. The $R^{2}$ measure is normalized to a value from 0.0 to 1.0 , with an optimal $R^{2}$ value of 1.0 meaning that the resulting fit curve lies directly on the data. The forms of the equations tested were the linear form, second-order polynomial, third-order polynomial, and the power function. Higher-order polynomial functions were attempted, but the statistical data-fit did not improve very much because the higher-order terms did not add a significant contribution to the curve shape.

Table 1 shows the results from fitting the different order functions through the pull-test sample data recovered for single wire minimum area and single wire short axis secondmoment diameter. Results are the minimum range capacity (for when the area goes to $0.0 \mathrm{~mm}^{2}$ and the minimum wire diameter goes to $0.0 \mathrm{~mm}$ ), the maximum range capacity (for pristine cable with area $20.27 \mathrm{~mm}^{2}\left(0.7980 \mathrm{in}^{2}\right.$ ) and smallest pristine (outer) wire diameter of $5.08 \mathrm{~mm}(0.2 \mathrm{in}$.)), and the $R^{2}$ measure of error. Of course, at the point where 
TABLE 1: Best-fit curve properties for single wire area and minimum second-moment short axis diameter, respectively.

\begin{tabular}{|c|c|c|c|c|c|c|}
\hline \multirow[b]{2}{*}{ Method } & \multicolumn{3}{|c|}{ Minimum wire area (all samples) } & \multicolumn{3}{|c|}{ Minimum wire diameter (all samples) } \\
\hline & $\begin{array}{l}\text { Min. area range } \\
\text { capacity for wire area } \\
\text { of } 0 \mathrm{~mm}^{2}(\mathrm{kN})\end{array}$ & $\begin{array}{l}\text { Max. area range capacity } \\
\text { for wire area of } \\
20.27 \mathrm{~mm}^{2}(\mathrm{kN})\end{array}$ & $R^{2}$ & $\begin{array}{c}\text { Min. diameter range } \\
\text { capacity for wire diameter } \\
\text { of } 0 \mathrm{~mm}(\mathrm{kN})\end{array}$ & $\begin{array}{c}\text { Max. diameter range } \\
\text { capacity for wire diameter } \\
\text { of } 5.08 \mathrm{~mm}(\mathrm{kN})\end{array}$ & $R^{2}$ \\
\hline Linear & 66.109 & 297.35 & 0.85 & -6.19 & 286.67 & 0.89 \\
\hline $\begin{array}{l}\text { Poly } \\
\text { Order } 2\end{array}$ & 18.75 & 273.66 & 0.9 & -39.54 & 279.13 & 0.89 \\
\hline $\begin{array}{l}\text { Poly } \\
\text { Order } 3\end{array}$ & 19.73 & 273.94 & 0.9 & 44.12 & 273.84 & 0.9 \\
\hline Power & 0 & 291.95 & 0.9 & 0 & 291.63 & 0.87 \\
\hline
\end{tabular}

the area of the cable is $0.0 \mathrm{~mm}^{2}$ or its minimum diameter is $0.0 \mathrm{~mm}$, the function to calculate cable strength should return a value approaching $0.0 \mathrm{kN}$ and at the point of maximum area and smallest pristine diameter should return a value in line with the average pristine pull-test results $274.451 \mathrm{kN}(61,699 \mathrm{lbf})$. It is extremely likely that the other wires in the cable are also corroded for the case where the minimum wire area is $0.0 \mathrm{~mm}^{2}$ and the minimum wire diameter is $0.0 \mathrm{~mm}$, and so the capacity is severely limited and approaches $0.0 \mathrm{kN}$. An insight gained from this table is that some of these equations, while having marginally better $R^{2}$ values, are better at predicting the end-point data.

Examining Table 1, the functions that best estimate the data including the end points are the polynomial order 2 equation for the minimum wire area (blue line in Figure 8) and the polynomial order 3 equation for the minimum wire diameter (blue line in Figure 9). These mean estimate functions are given as follows:

$$
y=-0.4605 x^{2}+21.901 x+18.574
$$

for the minimum wire area calculation, where $y$ is the capacity $(\mathrm{kN})$ and $x$ is the minimum wire area $\mathrm{mm}^{2}$, and

$$
y=-3.3709 x^{3}+29.217 x^{2}-16.247 x+44.12
$$

for the minimum wire diameter calculation, where $y$ is the capacity $(\mathrm{kN})$ and $x$ is the minimum wire diameter $(\mathrm{mm})$.

Figures 8 and 9 show the statistical data based on the selected trendlines for the minimum wire area and minimum wire diameter. For each trendline equation, the standard error for the capacity (along the force axis) was determined.

The standard error for the capacity of the wire given the minimum wire area is

$$
\mathrm{SE}_{\text {MinWireArea }}=\sqrt{\frac{\sum_{1}^{\text {numsamples }}\left[\text { Capacity }_{\text {sample }}-\text { Predictor }_{\left.(\text {Equation }(1))\left(\text { MinWireArea }_{\text {sample }}\right)\right]^{2}}\right.}{\text { numsamples }-1}},
$$

and the standard error for the capacity of the wire given the minimum wire diameter is

$$
\mathrm{SE}_{\text {MinWireDiameter }}=\sqrt{\frac{\sum_{1}^{\text {numsamples }}\left[\text { Capacity }_{\text {sample }}-\text { Predictor }_{\left(\text {Equation (2)) }\left(\text { MinWireDiameter }_{\text {sample }}\right)\right]^{2}}\right.}{\text { numsamples }-1}} .
$$

For the selected minimum wire area trendline equation, the samples had a standard error (or standard deviation) of $20.271 \mathrm{kN}$ ( $4557 \mathrm{lbf}$ ). For the selected minimum wire diameter trendline equation, the samples had a standard error of $20.413 \mathrm{kN}$ (4589 lbf). These standard errors vary by less than $1 \%$ from each other and suggest that either data-fit trendline would be appropriate for predicting the capacity of a cable given the minimum cable area for the first trendline equation and given the minimum wire diameter for the second trendline equation.
In Figures 8 and 9, the green lines portray the $\pm 2 \sigma$ values for the trendlines, and the $\pm 1 \sigma$ values are shown in the tan lines. The black lines in these figures give the $95 \%$ value, where $95 \%$ of the values lay above that line, from the CDF.

Additional confirmation of the trendline values can be established by realizing that the mean trendline curve is an increasing function with a peak capacity that does not exceed the pristine condition wires, or a minimum capacity with negative values. In fact, the mean trendline nearly passes through the center of the pristine cable samples at the 


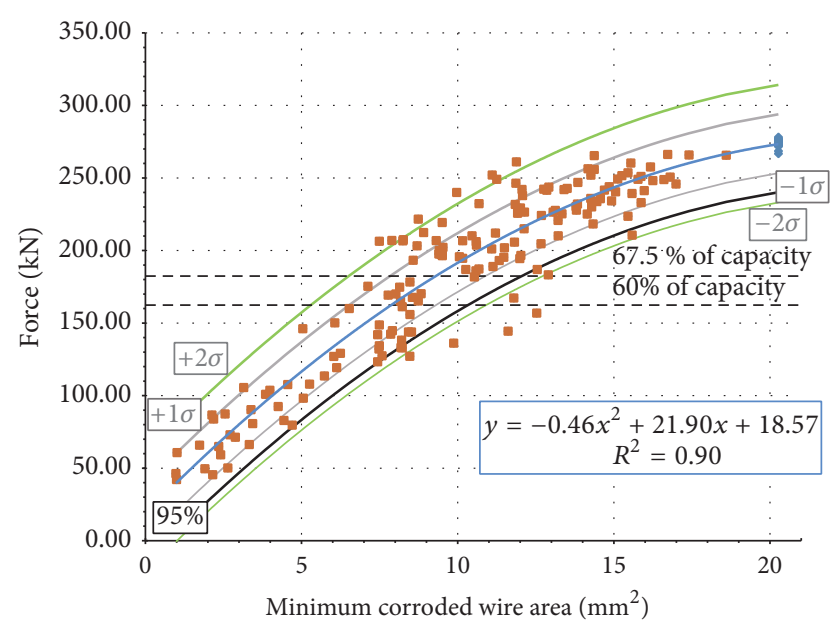

Figure 8: The selected data trendline (polynomial order 2) for the minimum wire area data-fit, its standard deviation $(\sigma)$ information, and the $95 \%$ cumulative distribution function (CDF) level information from data collected in Ebeling et al. [3]. Dashed lines represent potential relaxation limits of expected field load levels.

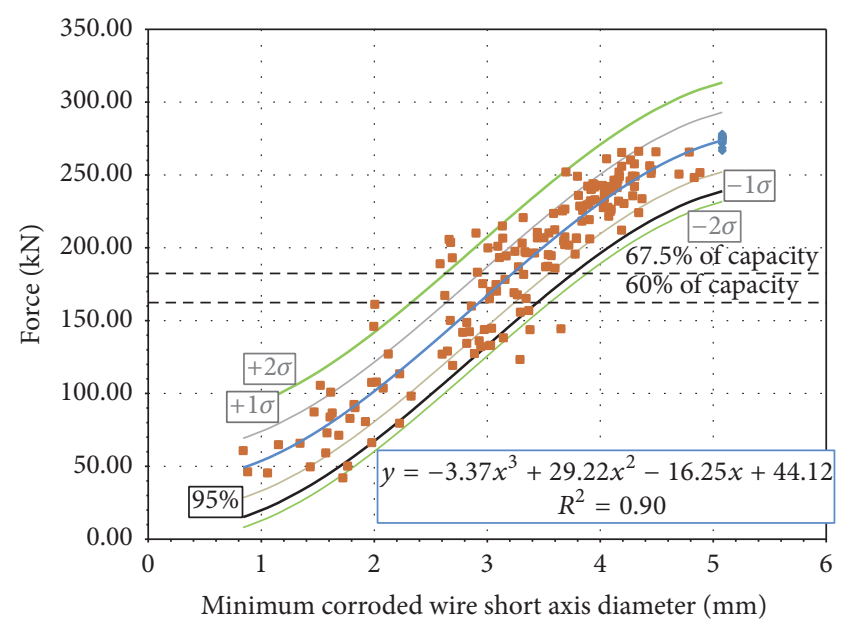

FIgURE 9: The selected data trendline (polynomial order 3) for the minimum wire diameter data-fit, its standard deviation $(\sigma)$ information, and the $95 \%$ CDF level information from data collected in [3].

greatest area and diameter in Figures 8 and 9, respectively. A slight concern about the curves is due to the fact that the equations for these curves overpredict the strength of the cables when the minimum wire area and minimum wire short axis diameter have a value of $0.0\left(\mathrm{~mm}^{2}\right.$ and $\mathrm{mm}$, resp.), which indicate that the capacity of the cable approaches 0.0 in $\mathrm{kN}$ and kips, respectively. As recall, it is extremely likely that the other wires in the cable are also corroded and in this case the capacity is severely limited and approaches $0.0 \mathrm{kN}$. These values are given as an offset of $y$ in the mean estimate functions of (1) and (2). For the minimum wire area calculation (see (3)), the value determined for a minimum wire area of $0.0 \mathrm{~mm}^{2}$ gives a capacity of $66.167 \mathrm{kN}$ (14.875 kips). For the minimum wire short axis diameter calculation (see (4)), the value determined for a minimum wire short axis diameter of $0.0 \mathrm{~mm}$ gives a capacity of $196.256 \mathrm{kN}$ (44.12 kips). Given the typical range of the loads that are applied to the multistrand anchors in the field (as indicated by the dashed black lines in Figures 8 and 9), it is felt that the significant range of allowable cable corrosion is covered well by the trendline equations.

2.5. A Method to Relate Test Data to Field Performance. The tensile testing process used here for the characterization of cable capacity as a function of cross section differs from that of an in situ cable failure process. Laboratory pull-testing of unconfined (i.e., ungrouted) cables was performed by pulling at a constant rate until failure. In mass concrete, the cables experience only minor load variations about their PT levels. Failure occurs in the field when the cross section of the smallest wire reaches a critically small level through corrosioninduced material losses. What happens after a wire fails is then dependent on the details of the installation. In bonded PT systems, a confining grout will serve to prevent cable distressing, allowing adjacent cables residual load-carrying capability without significant restraining. In unbonded systems, the cable will tend to unwind and the six remaining cables will occupy some of the volume once consumed by the broken strand. This unwinding process will shed a significant amount of load, the extent of unloading being a function of the unbonded gauge length. The unbonded PT case requires a large additional strain for the failed seven-strand assembly to begin carrying significant load again. For the failure of a bonded tendon, the impact is localized and the tendon will continue to contribute strength along its bonded length. So the remaining load carried by a seven-strand cable after a single wire fails is dependent on the bonding condition between the strand and surrounding concrete. The amount of increased load transfer to the adjoining strands in a multistrand anchorage once a failure occurs is dependent on the details of the anchorage system and the nature of its structural loading.

It was also observed in our laboratory pull-testing of unbonded cables that the failure of the smallest strand sometimes immediately leads to failure of nearby strands as the applied load was rebalanced among the remaining wires. These secondary failures are also likely affected by the reverberating energy between the anchorage ends. In the field, ejected king wires have been observed [10] where the propagating kinetic energy after failure literally ejects failed wires from their gripping wedges.

In situ stress levels are difficult to estimate and only measureable in certain scenarios. For example, data recovered from a lift-off test of an entire 37-strand anchor head at John Day Lock and Dam was used on several occasions to estimate collective remaining tension levels in the anchor heads [10]. A number of factors affect actual PT levels of in situ anchorages. These factors include operator and equipment error in setting initial levels; iterative tensioning of multistrand anchorages; relaxation of the steel cable; creep and shrinkage of the concrete; thermal and load variations. These factors, detailed in numerous reports, often depend on the aspects of the structural element and site-specific variables. In a retrofit scenario of older concrete structures, the reduction of initial 
stress is assumed for this article to be in the range of $2.5-10 \%$ for low relaxation cable. Estimating additional losses of 2.5$10 \%$ due to creep and other factors, the nominally applied $70 \%$ of capacity load leads to resulting load levels for the $15.24 \mathrm{~mm}(0.6 \mathrm{in}$.) diameter strand of 162.36 to $182.377 \mathrm{kN}$ (36,500 to $41,000 \mathrm{lbf})$. Since field failures generally occur when cable cross sections become critical for the applied load levels, a dashed line bounding region has been superimposed on the cross section versus ultimate tensile strength data in Figures 8 and 9; the upper dashed lines in each figure correspond to $67.5 \%$ of capacity load and the bottom dashed lines correspond to $60 \%$ of capacity load. The intersection of the mean wire area and mean wire diameter (from Figures 8 and 9, resp.) with the range of expected in situ load levels can be used as a means of estimating the critical area and diameter where in-service cables will fail. From the data in Figure 8, the minimum wire area at expected field loads will on average be $7.87 \mathrm{~mm}^{2}\left(0.0122 \mathrm{in}^{2}\right)$ to $9.42 \mathrm{~mm}^{2}\left(0.0146 \mathrm{in}^{2}\right)$. From Figure 9, the diameters will on average range from $2.92 \mathrm{~mm}(0.115 \mathrm{in}$.) to $3.23 \mathrm{~mm}(0.127 \mathrm{in}$.) at failure.

The green curves shown in Figure 9 represent two standard deviations from the mean minimum diameter. They are used to determine the minimum and maximum cable diameters for failure at the expected $60 \%$ of capacity load level (due to long-term losses from creep, etc., discussed above). This load level is depicted by the lower dashed black line of Figure 9. This produces minimum diameters of $3.626 \mathrm{~mm}(0.1427 \mathrm{in}$.) for $-2 \sigma$ and $2.406 \mathrm{~mm}$ (0.0947 in.) for $+2 \sigma$ from the mean. These values are subtracted from the original cable diameter of $5.08 \mathrm{~mm}(0.2 \mathrm{in}$.), resulting in expected minimum and maximum section losses of $1.454 \mathrm{~mm}$ (0.0572 in.) and $2.674 \mathrm{~mm}(0.1052 \mathrm{in}$.) for the $-2 \sigma$ and $+2 \sigma$ curves, respectively. This same procedure can be used to interpret mean value for the area section losses in Figure 8.

Ebeling et al. [3] provide a discussion of corrosion rates of steel in various environments. Corrosion loss rate is specified in either current density or incursion depth per year [3]. For metric units, the incursion loss is measured in microns/year (0.03937 mils/year). From Figure 9, the maximum diameter of the corroded cable before failure can be determined from the intersection of the $67.5 \%$ of capacity load line and the mean $-2 \sigma$ curve, resulting in a value of $3.87 \mathrm{~mm}(0.1523 \mathrm{in}$.). This corresponds to a $1.21 \mathrm{~mm}$ ( $0.0476 \mathrm{in}$.) diameter loss from the $5.08 \mathrm{~mm}(0.2 \mathrm{in}$.) cable. The same method can be used to find the minimum corroded diameter before failure from the intersection of the $60 \%$ of capacity load line and mean $+2 \sigma$ curve, resulting in a value of $2.32 \mathrm{~mm}$ (0.0913 in.). This corresponds to a $2.76 \mathrm{~mm}$ ( $0.1086 \mathrm{in}$.) diameter loss. Figure 10 provides a rough indication of the maximum and minimum time in years to cable failure as a function of loss rate in microns per year, using the diameter loss values of $1.21 \mathrm{~mm}$ (0.0476 in.), blue curve, and $2.76 \mathrm{~mm}$ (0.1086 in.), red curve.

\section{Conclusions}

Multistrand anchors, based on stressed steel tendons, provide strength and stability to mass concrete hydraulic structures. These steel tendons are subject to strength reduction as a result of pitting corrosion. A way to evaluate the reduced

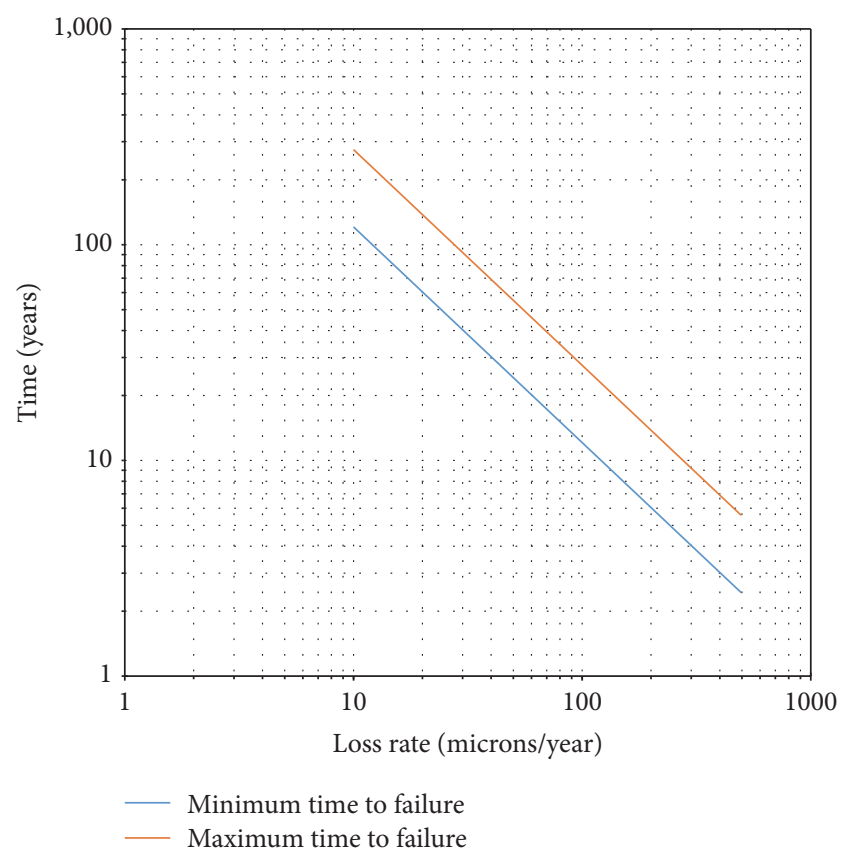

FIGURE 10: Estimated minimum and maximum time to failure as a function of corrosion loss rate (from data collected and analyzed in Ebeling et al. [3]).

strength of these cables is needed and could lead to an estimate of time until these cables need to be replaced. Methods for performing laboratory accelerated corrosion, pull-tests on pristine and laboratory corroded cables, optical scanning, and data collection for cross-sectional properties of cables (i.e., area, minimum second-moment diameter) have been discussed. Pull-tests provided peak capacity of each pristine and corroded seven-strand, PT cable. Likewise, the optical scan of the corroded cables provided the cross section properties of the cables and individual wires. Trendlines were established for the related peak capacities and cross-sectional properties. The trendline for peak capacity and minimum wire area had a very high $R^{2}$ value of 0.9 for an order 2 polynomial function. The trendline for peak capacity and minimum wire second-moment short axis diameter had a very high $R^{2}$ value of 0.9 for an order 3 polynomial function. The high $R^{2}$ values indicate that these trendlines are good predictors for peak cable capacity. These data were related back to cable failure in the field, assuming a linear rate of corrosion loss for the cross-sectional properties and required PT capacity.

From this effort, it is concluded that the strength of a seven-strand wire cable is directly related to the strength of the individual strands that are wound about the king wire. Using artificial corrosion and optical scanning techniques, attributes of corroded individual strands were computed. The primary attributes that correlate to the capacity of corroded individual strands are the reduced cross-sectional area and the minimum second-moment diameter. Given a corrosion rate and exposure time for an installation, the time of failure of cables in a tendon system can be estimated using the statistical trends established in the paper. 


\section{Competing Interests}

The authors declare that there is no conflict of interests regarding the publication of this paper.

\section{Acknowledgments}

Funds to perform this work were provided by the Navigation Systems Research Program of the U.S. Army Corps of Engineers.

\section{References}

[1] A. Azizinamini and J. Gull, "Improved inspections techniques for steel pre-stressing/post tensioning strand," FDOT Contract BDK80 977-13, Final Report-Volume 1, Florida International University, Miami, University Park, Miami, Fla, USA, 2012, http://www.dot.state.fl.us/research-center/Completed_Proj/ Summary_MNT/FDOT-BDK80-977-13-rptvoll.pdf.

[2] I. Bartoli, S. Salamone, R. Phillips, C. Nucera, and F. Scalea, "Health monitoring to detect failure of prestressing (PS) cables in segmental box-girder bridges," Tech. Rep. SSRP 0906, Department of Structural Engineering, University of California, San Diego, San Diego, Calif, USA, 2012.

[3] R. M. Ebeling, B. C. White, J. A. Evans, R. W. Haskins, and E. L. Miller, "Corrosion induced loss of capacity of post-tensioned seven wire strand cable used in multistrand anchor systems installed at corps projects," Tech. Rep. ERDC/ITL TR-16-4, Engineering Research and Development Center, Vicksburg, Miss, USA, 2016.

[4] S.-K. Lee and J. Zielske, "An FHWA special study: post-tensioning tendon grout chloride thresholds," Federal Highway Administration Report FHWA-HRT-14-039, Research, Development, and Technology Turner-Fairbank Highway Research Center, McLean, Va, USA, 2014.

[5] T. Hopwood and J. H. Havens, "Corrosion of cable suspension bridges," Research Report UKTRP-84-14, Kentucky Transportation Research Program, College of Engineering, University of Kentucky, Lexington, Ky, USA, 1984, http://www.ktc .uky.edu/files/2012/09/1984-Corrosion-of-Cable-SuspensionBridges-UKTRP-84-14.pdf.

[6] M. S. Darmawan and M. G. Stewart, "Effect of pitting corrosion on capacity of prestressing wires," Magazine of Concrete Research, vol. 59, no. 2, pp. 131-139, 2007.

[7] D. Trejo, R. G. Pillai, M. B. D. Hueste, K. F. Reinschmidt, and P. Gardoni, "Parameters influencing corrosion and tension capacity of post-tensioning strands," ACI Materials Journal, vol. 106, no. 2, pp. 144-153, 2009.

[8] R. D. Moser, High-strength stainless steels for corrosion mitigation in prestressed concrete: development and evaluation [Ph.D. thesis], Georgia Institute of Technology, Atlanta, Ga, USA, 2011.

[9] E. Dougherty, An Introduction to Morphological Image Processing, SPIE Optical Engineering Press, Bellingham, Wash, USA, 1992.

[10] R. M. Ebeling, R. W. Strom, J. E. Hite, R. W. Haskins, and J. A. Evans, Assessing Corrosion Damage and Corrosion Progression in Multistrand Anchor System in Use at Corps Projects, ERDC TR-13-3, Engineering Research and Development Center, Vicksburg, Miss, USA, 2013, http://acwc.sdp.sirsi.net/client/default/index.assetbox.assetactionicon.view/1028160;jsessionid= 755CB8E9AB17854C8D2B54CE9D0D9FFC.enterprise-15000? rm=ENGINEER+RESEA0\%7C\%7C\%7C1\%7C\%7C\%7C0\% 7C\%7C\%7Ctrue. 


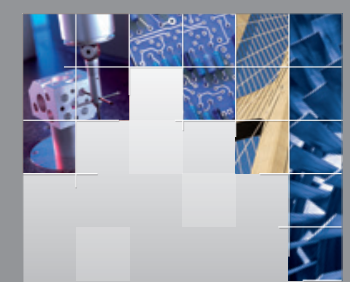

\section{Enfincering}
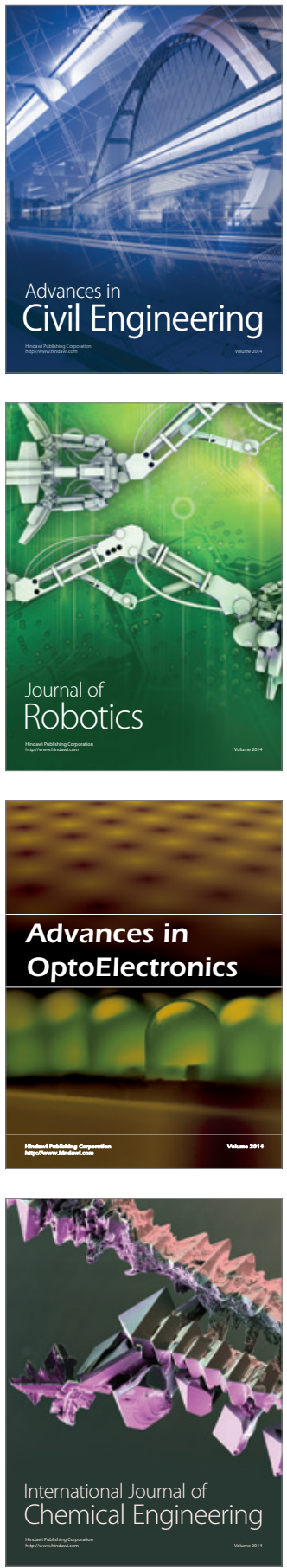

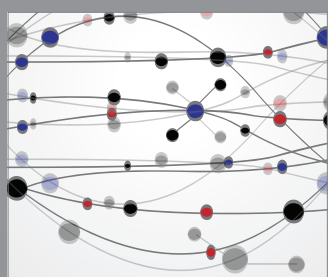

The Scientific World Journal

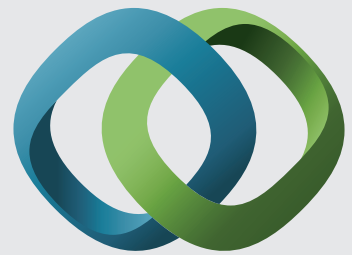

\section{Hindawi}

Submit your manuscripts at

http://www.hindawi.com
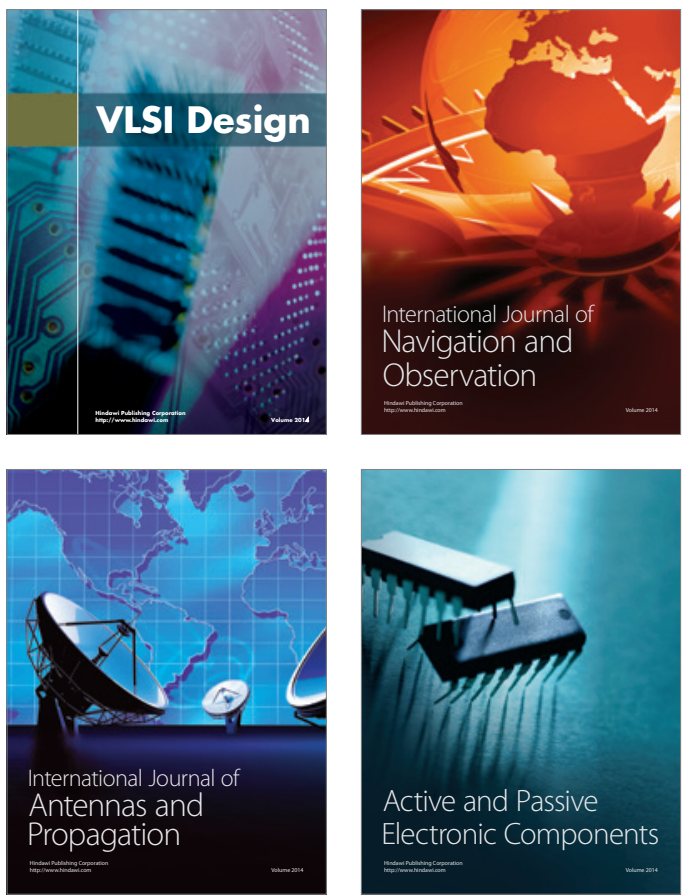
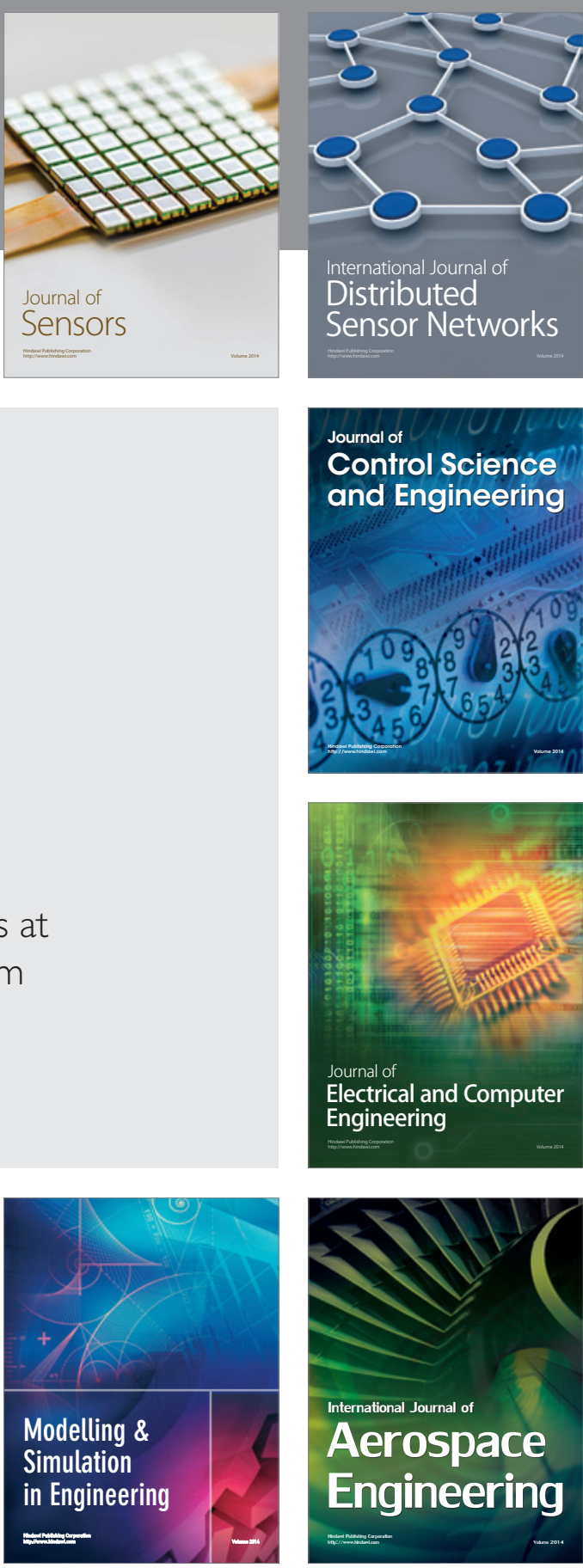

International Journal of

Distributed

Sensor Networks

Journal of

Control Science

and Engineering
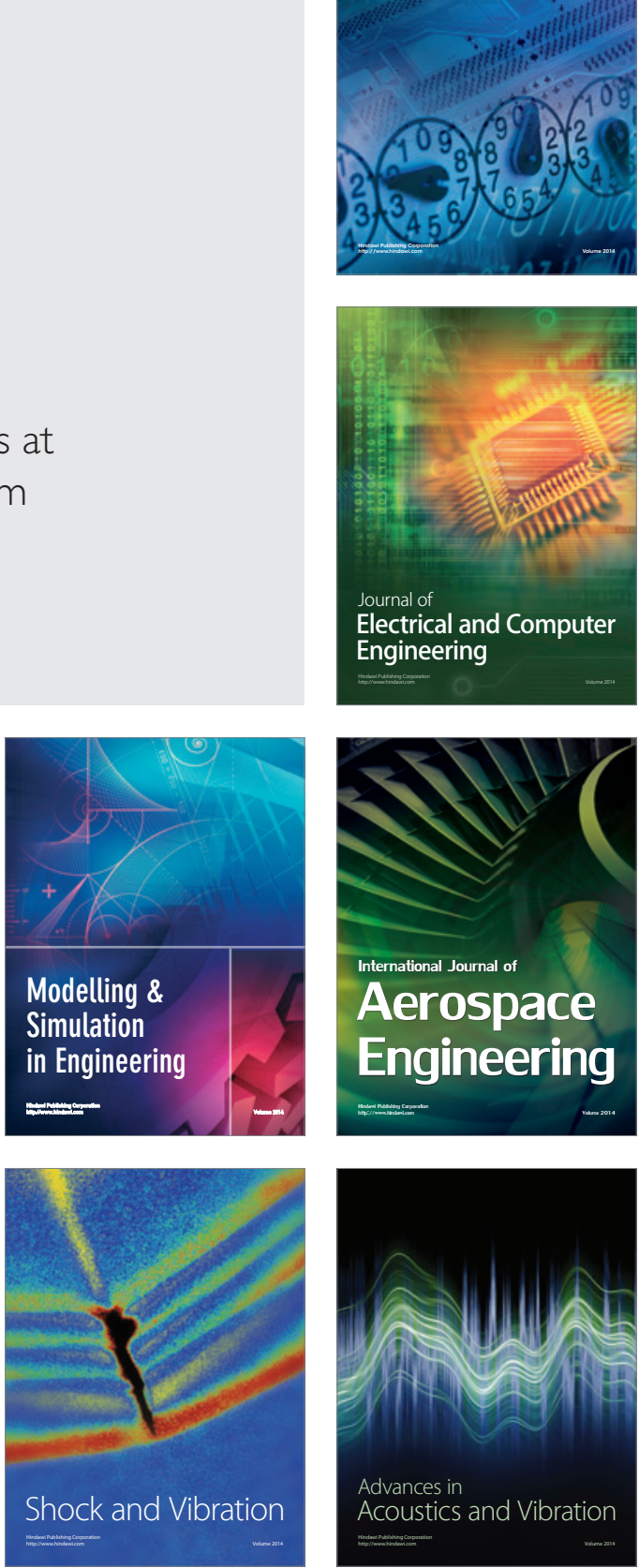